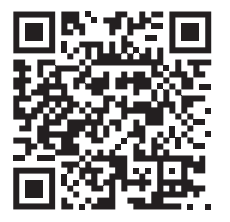

* Maestro en Ciencias Profesor de Asignatura, Investigador independiente. ‡ Lic. Químico Farmacéutico Biotecnólogo, Escuela de Ciencias de la Salud, Universidad del Valle de México campus Coyoacán, Ciudad de México.

$\S$ Estudiante de la licenciatura en Químico Farmacéutico Biotecnólogo.

Correspondencia: EMV eduardomucito@gmail. com

Conflicto de intereses: Los autores declaran no tener.

Citar como: MucitoVarela E, Sánchez-Oviedo FV. Tecnovigilancia en los laboratorios clínicos: una herramienta para la seguridad del paciente. Rev CONAMED. 2020; 25(4): 174-181. https://dx.doi. org/10.35366/97337

Financiamiento: Ninguno.

Recibido: 12/10/2020

Aceptado: 24/11/2020

\section{Tecnovigilancia en los laboratorios clínicos: una herramienta para la seguridad del paciente}

\author{
Technovigilance in clinical laboratories: a tool for patient safety \\ Eduardo Mucito-Varela, , Fernanda Vanessa Sánchez-Oviedo $\ddagger, \S$
}

\section{RESUMEN}

La atención sanitaria integra el uso de dispositivos médicos durante sus procesos, los cuales deben cumplir con las características de calidad y desempeño establecidas en la normatividad oficial de cada país. La tecnovigilancia es una herramienta que permite garantizar la seguridad de los pacientes al facilitar la notificación de eventos adversos asociados al uso de dispositivos médicos. Los laboratorios clínicos, como usuarios de dispositivos médicos, no están exentos de la tecnovigilancia, por lo que deben alinear los requisitos de ésta con los requerimientos particulares de funcionamiento y de gestión de la calidad. En esta revisión se discute la importancia de la tecnovigilancia y se analiza el marco regulatorio para la instauración de la misma en los laboratorios clínicos de México.

Palabras clave: Tecnovigilancia, seguridad del paciente, dispositivos médicos, gestión de riesgos, laboratorio clínico.

\begin{abstract}
Health care includes the use of medical devices during its processes, which must comply with the quality and performance characteristics established in the official regulations of each country. Technovigilance is a tool that allows assurance of patient safety by facilitating the notification of adverse events associated with the use of medical devices. Clinical laboratories as users of medical devices are not exempt from technovigilance, so they must align the requirements of this with their particular operational and quality management requirements. This review discusses the importance of technovigilance and analyzes the regulatory framework for its implementation in clinical laboratories of Mexico.
\end{abstract}

Keywords: Technovigilance, patient safety, medical devices, risk management, clinical laboratory. 


\section{INTRODUCCIÓN}

La seguridad del paciente se logra por un conjunto de procesos que las organizaciones prestadoras de servicios de salud planean, ejecutan y monitorean en todos sus niveles para disminuir el riesgo de que los pacientes no sufran daños innecesarios durante la atención.' Dado que los servicios de salud son procesos complejos que integran a diferentes profesionales, que a su vez utilizan variedad de tecnologías con su complejidad individual, el riesgo de ocasionar daño al paciente siempre está latente.² Las tecnologías empleadas por los profesionales de la salud se consideran como «dispositivos médicos», que incluyen todos los aparatos, instrumentos o máquinas que son herramientas utilizadas para el diagnóstico, prevención o tratamiento de enfermedades; entre los que se incluyen kits de detección y pruebas rápidas, equipos de laboratorio e imagen, software de equipo, material de curación, catéteres y soluciones desinfectantes. ${ }^{3}$

Las metas internacionales de seguridad del paciente son los ejes rectores para su seguridad en los servicios de salud, las cuales, en México, permitieron la implementación obligatoria de las "Acciones esenciales para la seguridad del paciente». ${ }^{4}$ Dentro de estas acciones, la «seguridad en los procedimientos» y el «Registro y análisis de eventos centinela, eventos adversos y cuasi fallas» plantean la necesidad de monitorear el uso apropiado y funcionamiento correcto de los dispositivos médicos para detectar incidentes derivados del uso de los mismos con potencial de causar daño (cuasi fallas), que causaron daño (eventos adversos) o que causaron la muerte del paciente (eventos centinela).

La tecnovigilancia es un conjunto de procedimientos que garantizan que los dispositivos médicos disponibles en el mercado sean utilizados de acuerdo con la intención de uso indicada por el fabricante, que cumplan con las especificaciones de calidad y funcionalidad señaladas en la normatividad aplicable, así como en lo estipulado en la autorización sanitaria emitida por la Secretaría de Salud para el dispositivo; y que, en dado caso de que se identifique un incumplimiento, error o incidente, se realice una evaluación de riesgo y se establezcan las acciones correctivas que se consideren pertinentes para disminuir la recurrencia del evento reportado. ${ }^{5}$
En México existe el Centro Nacional de Farmacovigilancia (CNFV) que en conjunto con la Comisión Federal para la Protección contra Riesgos Sanitarios, recibe y recopila los reportes de las sospechas de Reacciones Adversas de Medicamentos, vacunas y dispositivos médicos por parte de los integrantes de la farmacovigilancia en el país, así como la evaluación, el análisis y la retroalimentación de la información, a partir de la cual realizan propuestas de políticas, programas y procedimientos para emitir un dictamen de acciones preventivas y correctivas que debe cumplir el fabricante. ${ }^{6}$

El objetivo de la presente revisión es conocer el estado del arte sobre la tecnovigilancia de los dispositivos médicos y su relación con la seguridad del paciente en el ámbito del laboratorio clínico y medicina de laboratorio.

\section{Eventos adversos asociados al uso de dispositivos médicos en la medicina de laboratorio}

Los laboratorios clínicos durante su proceso de operación, también denominado proceso total de examen, son usuarios de dispositivos médicos, por lo que no están exentos de la presencia de fallas que puede ocasionar un incidente que afecte la seguridad del paciente, causando daño directo o indirecto a través de errores de laboratorio que conduzcan a errores médicos (Figura 7). Actualmente, se desconoce la frecuencia real de incidentes asociados al uso de dispositivos médicos en nuestro país, según las estadísticas publicadas por la Comisión Federal para la Protección contra Riesgos Sanitarios (COFEPRIS) en 2018. Se registraron 2,140 incidentes en el periodo comprendido entre 2014y 2018, los cuales se resumen por tipo de dispositivo en la Tabla $7 .^{7}$

Los modos de fallo o errores de los dispositivos médicos son diversos y dependen de la naturaleza y complejidad de los mismos (Tabla 2). Estos modos de fallo o errores del dispositivo pueden originar errores de laboratorio y médicos que comprometan la seguridad del paciente, pero también pueden ocasionar daño directo al paciente durante el proceso de atención (Figura 7). Sin embargo, existen pocos reportes sobre los fallos de los dispositivos médicos e incidentes originados durante su uso en los laboratorios clínicos. 


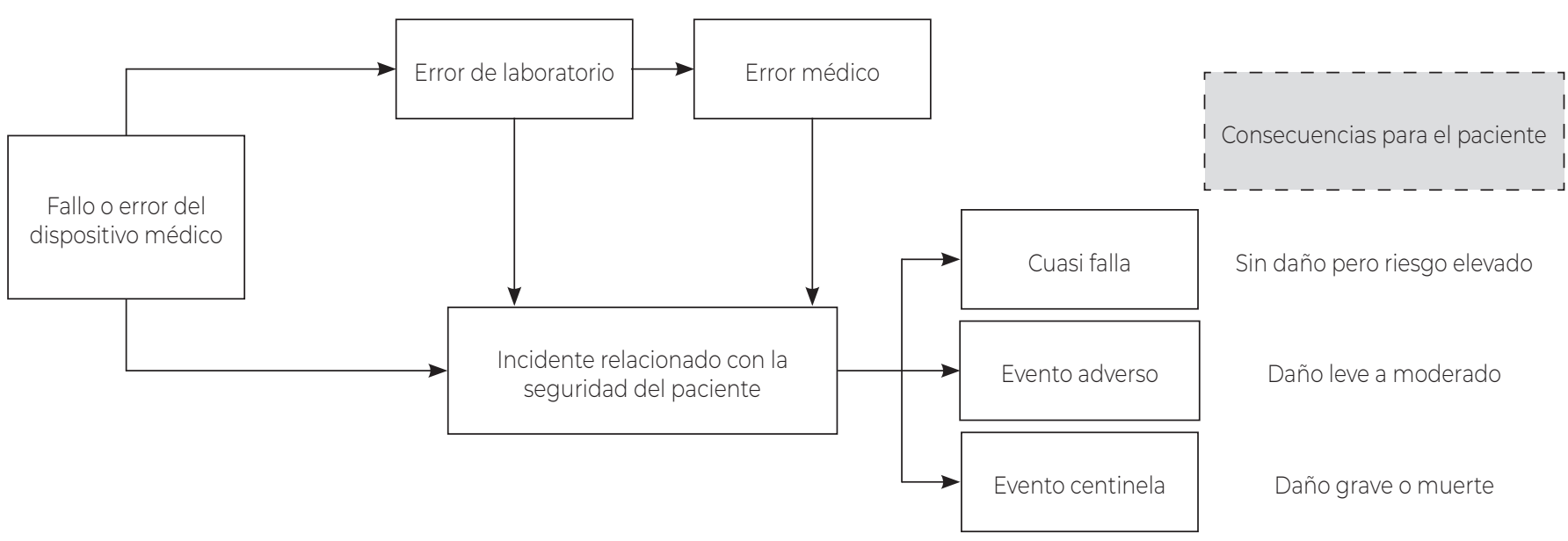

Figura 1: Uso de dispositivos médicos y su impacto en la seguridad del paciente. Las fallas o errores de origen de los dispositivos médicos pueden contribuir a la generación de incidentes con diferentes consecuencias para el paciente, siendo los eventos adversos y eventos centinela los de mayor impacto en la salud del paciente. Los incidentes se pueden generar cuando se usa directamente el dispositivo médico en el paciente (p.e. agujas, espejos vaginales, medio de contraste, pruebas rápidas), pero también cuando se utiliza con muestras derivadas de él (ella) originando «errores de laboratorio», que a su vez pueden generar «errores médicos» como retraso en el diagnóstico y/o tratamiento (p.e. uso inapropiado, interferencia analítica, errores de medición, mala validación del fabricante, pérdida de datos en el software).

Tabla 1: Número de incidentes asociados al uso de dispositivos médicos reportados a la COFEPRIS en el periodo 2014-2018.

\begin{tabular}{lc}
\hline Tipo de dispositivo & $\begin{array}{c}\text { Número de } \\
\text { incidentes } \\
\text { reportados }\end{array}$ \\
\hline Equipo médico & 667 \\
Materiales quirúrgicos y de curación & 617 \\
Prótesis & 236 \\
Ayuda funcional & 210 \\
Agentes de diagnóstico & 160 \\
Insumos de uso odontológico & 154 \\
Otro & 50 \\
Producto higiénico & 42 \\
Instrumental médico & 4 \\
Total & 2,140 \\
\hline
\end{tabular}

Comisión Federal para la Protección contra Riesgos Sanitarios. Información Referente a Tecnovigilancia 2014-2018. Gobierno de México; 2018. Disponible en: https://www.gob. $\mathrm{mx} / \mathrm{cms} /$ uploads/attachment/file/432576/INCIDENTES_ RELATIVOS_A_DISPOSITIVOS_M_DICOS_CLASE_II_Y_III_RECIBIDOS_EN_EL_CNFV_DE_2014_A_LA_FECHA_2.1.pdf.
En el informe de la COFEPRIS antes mencionado, se pueden identificar cinco reportes de incidentes debidos al mal funcionamiento de las agujas de extracción de sangre para sistemas al vacío, lo que ocasionó tomas de muestra inapropiadas y punciones repetidas al paciente. Aunque estos reportes representan apenas el 0.8\% (5/617) de los incidentes asociados al uso de materiales quirúrgicos y de curación, probablemente sea una subestimación, ya que éstos podrían no reportarse, debido a que el riesgo de daño a los pacientes es bajo. Sin embargo, el daño al paciente implica cualquier alteración física o emocional, que por mínimo que parezca, a nivel individual puede tener un impacto psicológico importante. En cuanto al uso de agentes de diagnóstico, el incidente que se puede asociar a la medicina de laboratorio es la toxicidad a los medios de contraste con una frecuencia de $8.75 \%$ (14/160) en los incidentes de este rubro, y que la mayoría de los casos fue considerado un efecto adverso esperable con poco o ningún daño al paciente, pero cuatro casos presentaron anafilaxia.

Los tipos y frecuencias de ocurrencia de los incidentes asociados al uso de dispositivos médicos se deben investigar en cada laboratorio para conocer el impacto real en la seguridad 
del paciente. Cada etapa del proceso total de examen puede tener diferentes riesgos para la seguridad del paciente; por ejemplo, el proceso de toma de muestras podría ser considerado el más riesgoso de los procesos de la fase preexamen, en donde puede haber reacciones alérgicas a los materiales de los dispositivos como el látex. ${ }^{8}$ Las lesiones por mal funcionamiento o mal diseño del dispositivo durante procedimientos de toma de muestra no se descartan, como ruptura de agujas o lesiones por espejos vaginales que han ocurrido en otros contextos de atención a la salud.9,10

En la etapa de examen, la falla del sistema de medición (equipo, reactivos y software) es uno de los incidentes asociados al uso de dispositivos médicos que pueden ocurrir." También se pueden encontrar esporádicamente casos de interferencia de los materiales del dispositivo con el sistema de medición, Ilevando a errores de diagnóstico, que la mayoría de las veces resultan en cuasi fallas, debido a la detección oportuna, pero con un riesgo elevado de daño al paciente si no se hubieran detectado. ${ }^{12}$ Los falsos positivos o negativos en las pruebas moleculares también se pueden considerar como un incidente derivado del mal diseño y validación del método. ${ }^{13}$

\section{Instauración de la tecnovigilancia en el laboratorio clínico}

La gestión de los eventos adversos asociados al uso de dispositivos médicos es responsabilidad de quienes los producen, distribuyen, comercializan, manipulan, prescriben y usan. Por lo que los laboratorios clínicos no están exentos de la

Tabla 2: Modos de fallas de origen en los dispositivos médicos relacionados con la tecnovigilancia.

\begin{tabular}{ll}
\hline Categoría & Modo de falla \\
\hline Asociados al dispositivo & Fallo del dispositivo \\
& Error en el diseño o de ergonomía \\
& Error en el etiquetado \\
& Error de fabricación o ensamblaje \\
& Deficiencias en el software \\
& Faltas aleatorias de los componentes y accesorios \\
& Validación deficiente por el fabricante \\
& Equipo o dispositivo descalibrado de fábrica \\
& Materiales de fabricación inapropiados \\
& Instructivos poco claros o ausentes \\
& Poca información sobre la utilidad clínica \\
Asociados al uso & Poca información sobre interferencias de medición \\
& Especificaciones de desempeño previstas poco claras \\
& Falta de esquemas o diagramas que permitan identificar las partes y/o ensamblaje \\
& Tecnología que requiera de personal especializado \\
& Falta de especificaciones de infraestructura en el instructivo \\
& Falta de especificaciones ambientales de almacenamiento y operación en el instructivo \\
Interacción con factores de asesoría técnica durante la instalación \\
externos & Falta de especificaciones ambientales de transporte \\
Soporte y mantenimiento de soporte técnico inmediato \\
Falta de refacciones de acceso inmediato \\
\hline
\end{tabular}




\section{Ciclo de vida del dispositivo médico}

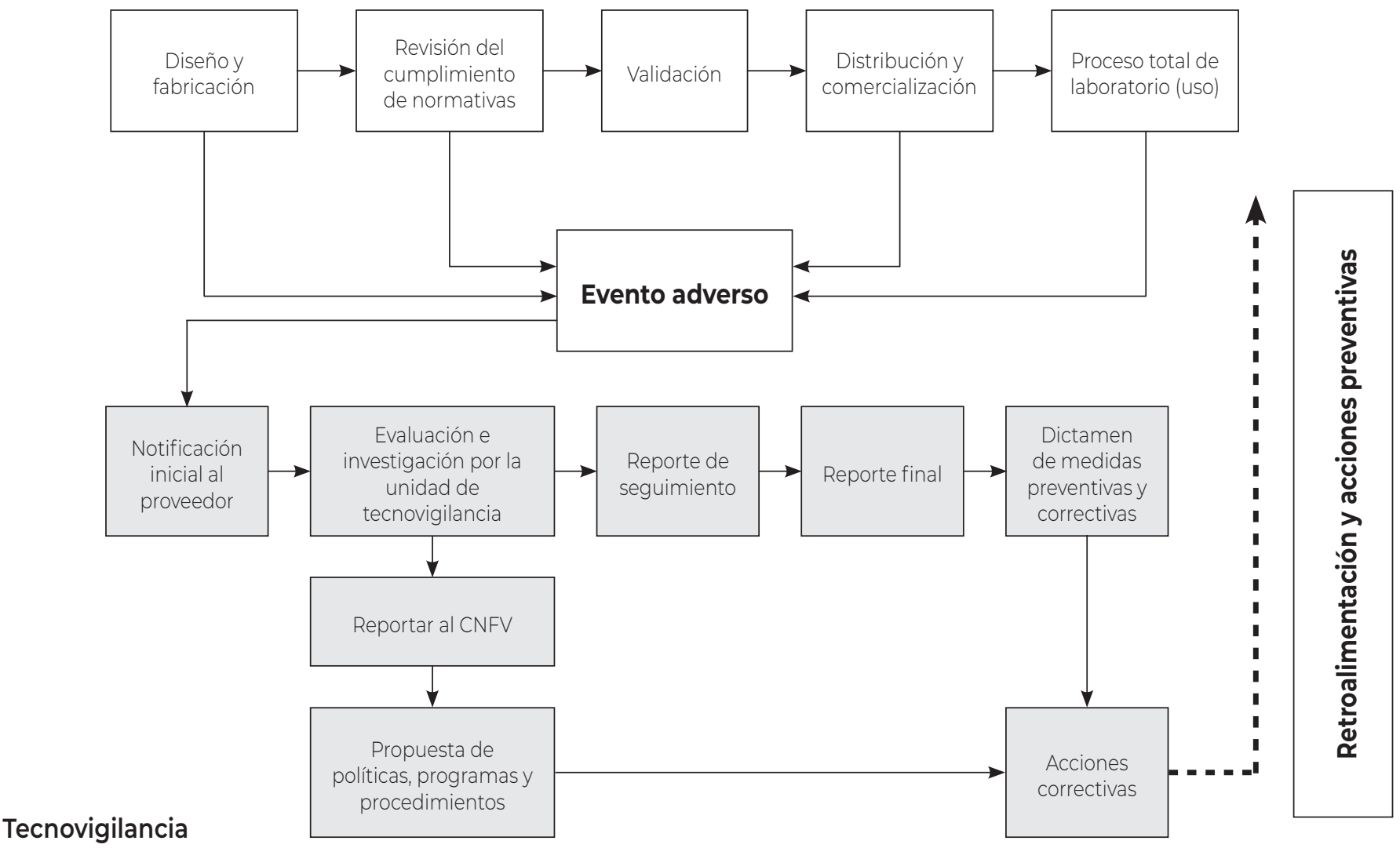

Figura 2: Proceso total de farmacovigilancia en el laboratorio clínico. El proceso de tecnovigilancia para dispositivos médicos inicia con el diseño y fabricación de éstos, en donde es necesario revisar el cumplimiento de las normativas vigentes y aplicables, así como los lineamientos emitidos por las autoridades sanitarias; posteriormente, el dispositivo pasa por validación para poderlo distribuir y comercializar, y por último, se utiliza durante el proceso total de laboratorio. Si en cualquiera de las fases se reporta un evento adverso, se requiere notificar al proveedor y realizar una evaluación e investigación por la unidad de tecnovigilancia, al mismo tiempo que se reporta al CNFV. La unidad de tecnovigilancia emite un reporte de seguimiento, a partir del cual se redacta un informe final y un dictamen de medidas preventivas y correctivas; que aunado a las propuestas de políticas, programas y procedimientos emitidos por la CNFV, permite establecer las acciones necesarias para el dispositivo en cuestión y mejorar la seguridad del dispositivo médico.

instauración de la tecnovigilancia. Es importante diferenciar entre los incidentes derivados del uso de dispositivos y aquellos relacionados con errores del proceso total de examen, ya que en estos últimos, el dispositivo no ha estado en contacto con los pacientes o sus muestras y derivan de factores externos no relacionados con ellos. ${ }^{5}$

El proceso que debe seguir el laboratorio clínico es el establecido por la Norma Oficial Mexicana NOM-240-SSA1-2012 que se resume en la Figura 2. A nivel operacional, la tecnovigilancia se puede alinear con el sistema de gestión de la calidad (Figura 3), ya que los principios fundamentales de calidad favorecen el entorno para la seguridad del paciente a través de la gestión de riesgos, la documentación para la notificación de errores e incidentes y la mejora de la seguridad de los dispositivos médicos.

\section{Gestión de riesgos}

La gestión de riesgos tiene como finalidad detectar fallas en los procesos antes de que éstas ocurran, aunque también permite aprender de los errores ya ocurridos, de manera que se puede considerar una estrategia proactiva-reactiva para la seguridad del paciente..$^{4,15}$ Las técnicas de gestión de riesgos 
para laboratorios clínicos se detallan en el EP18 del Clinical and Laboratory Standards Institute (CLSI) y se integran como herramientas del control de calidad planificado en el estándar EP23A, para detectar, prevenir y controlar errores que pueden causar daño al paciente durante el proceso total de examen. ${ }^{16}$ En la tecnovigilancia de los dispositivos médicos, las técnicas de gestión de riesgos se pueden aplicar durante el desarrollo y validación por el fabricante, así como la verificación y uso por el laboratorio clínico (Tabla 3).

\section{Notificación de errores e incidentes}

La investigación de errores de laboratorio e incidentes a través del análisis de la documentación del sistema de gestión de calidad (p.e. verificaciones de rutina, control de calidad, quejas y encuestas de satisfacción a usuarios, etcétera), permitirán definir la asociación de fallos en los dispositivos médicos con los eventos adversos para notificarlos al proveedor y se ejecuten los procesos de tecnovigilancia. Por lo que el laboratorio debe contar con los procedimientos estandarizados pertinentes que utilicen los medios establecidos por las autoridades sanitarias para este proceso de notificación, los cuales deberían formar parte del manual de procedimientos administrativos requeridos en la NOM-007-SSA3-2011 y de los requisitos de gestión de acuerdo con el modelo ISO 15189:2012.17,18 El incluir estas actividades en el laboratorio clínico permitirá avanzar hacia el cumplimiento de la acción esencial Registro y análisis de eventos centinela, eventos adversos y cuasi fallas. ${ }^{4}$

\section{Mejora de la seguridad de dispositivos}

La comunicación entre los proveedores y el laboratorio clínico puede contribuir a mejorar la seguridad de los dispositivos médicos, incluyendo el uso apropiado de los mismos. El laboratorio debe definir las especificaciones esperadas de un dispositivo médico de acuerdo con las necesidades de su proceso total de examen. Éstas se deben

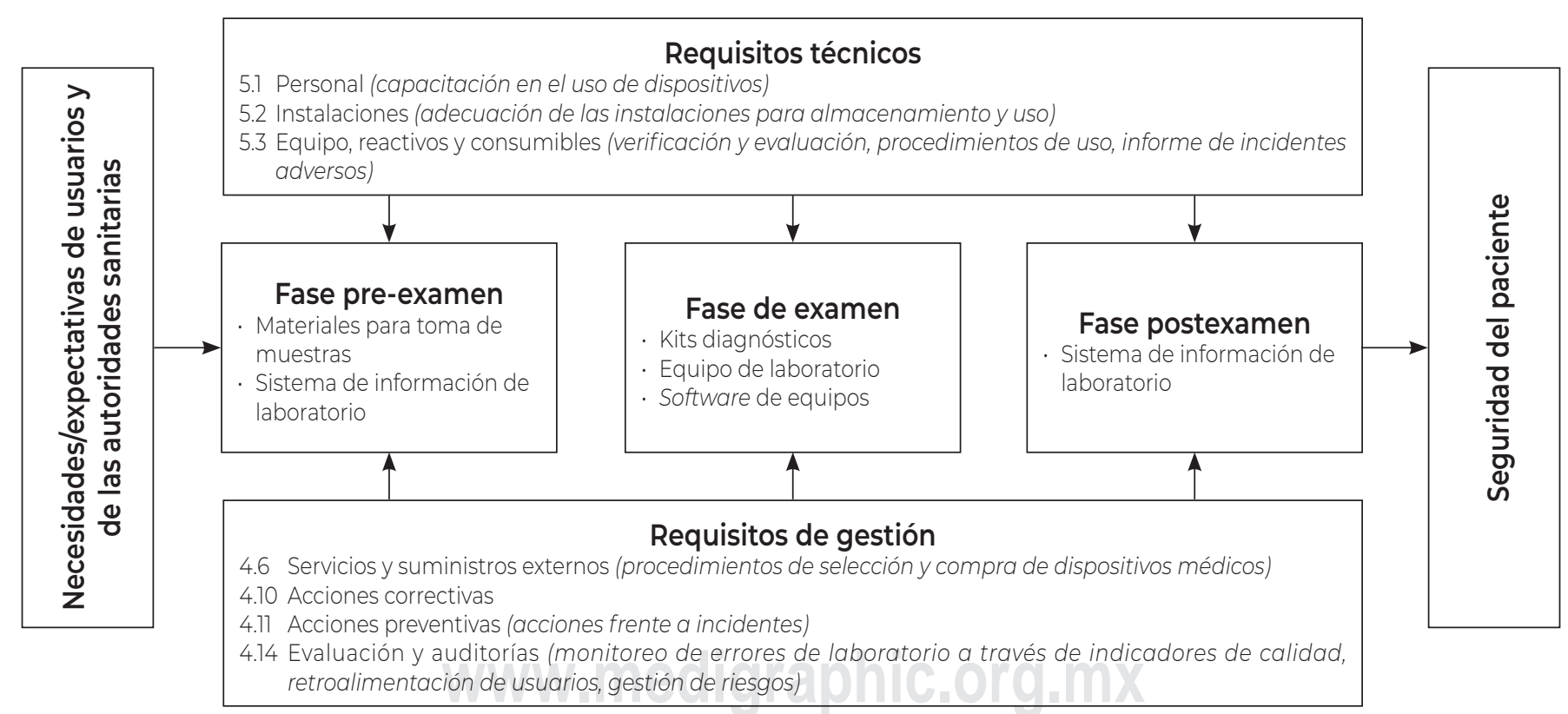

Figura 3: Alineación de la tecnovigilancia con el sistema de gestión de calidad de los laboratorios clínicos. Esquema del modelo de sistema de gestión de la calidad basado en la ISO 15189:2012, que establece requisitos particulares para los laboratorios clínicos. Se observa en cada etapa del proceso total de examen el uso de diferentes dispositivos médicos, los cuales son objeto de la tecnovigilancia. La instauración de la tecnovigilancia a través de las acciones enlistadas entre paréntesis, se sobreponen con los requisitos técnicos y de gestión de la norma. Esto permite lograr satisfacer las necesidades y expectativas de los usuarios de los servicios de laboratorio, pero también cubrir los requisitos regulatorios para la tecnovigilancia, siendo la seguridad del paciente el resultado final. 
Tabla 3: Uso de las herramientas de gestión de riesgos para la tecnovigilancia.

\begin{tabular}{lll}
\hline Herramientas & Características & Recomendación de uso en dispositivos médicos \\
\hline FMEA & Preventiva, proactiva & $\begin{array}{l}\text { Diseño del producto } \\
\text { Planificación del uso y control de calidad del dispositivo por el laboratorio } \\
\end{array}$ \\
FRACAS & Cerificación del producto por el laboratorio o usuario final \\
& & Validación y mejora de la prueba por el fabricante \\
& Detección de errores durante el uso del producto por el laboratorio \\
\hline
\end{tabular}

FMEA = análisis modal de fallos y efectos, FRACAS = análisis y gestión de acciones correctivas contra las fallas.

evaluar durante la selección y compra, como parte los requisitos de gestión del sistema de calidad; y se deben verificar antes de usar los dispositivos con los pacientes o sus muestras, bajo las condiciones ambientales y de infraestructura requeridas de acuerdo con las validaciones por el fabricante, esto forma parte de los requisitos técnicos del sistema de gestión. En los requisitos legales para los laboratorios en México, se deberían integrar en los manuales de procedimientos administrativosy en la bitácora de mantenimiento y calibración de equipo, respectivamente.

El conocimiento de estos requisitos esperados para el dispositivo médico, la evaluación del desempeño durante su uso, en conjunto con la notificación de errores e incidentes derivados del uso del mismo permitirá que la industria detecte oportunidades de mejora para sus procesos de investigación y desarrollo, favoreciendo el rediseño o producción de nuevas tecnologías. De esta manera, la industria de dispositivos médicos retroalimenta su inteligencia tecnológica, beneficiándose mutuamente con los servicios de salud. ${ }^{19}$

\section{CONCLUSIONES}

La tecnovigilancia contribuye a la seguridad del paciente a través de sus procesos de identificación y notificación de eventos adversos, lo que permite mejorar la seguridad de los dispositivos médicos. Los laboratorios clínicos cuentan en la actualidad con un marco regulatorio y de gestión de la calidad que se sobrepone con las actividades de la tecnovigilancia. Sin embargo, aún existen brechas para la instauración de la tecnovigilancia en los laboratorios clínicos; por ejemplo, la inclusión de procedimientos de evaluación inicial y la detección de eventos adversos asociados al uso de dispositivos médicos en los requisitos obligatorios de la NOM-007-SSA3-2011 para el funcionamiento de los laboratorios clínicos. Adicionalmente, la definición de responsabilidades para el responsable sanitario y el personal del laboratorio para la ejecución de los procesos de farmacovigilancia, es otro elemento que se debe cubrir en la normatividad aplicable a los laboratorios clínicos.

Oportunidades de mejora de la tecnovigilancia de manera general son la armonización de la terminología para la seguridad del paciente para realizar la correcta identificación, investigación, tipificación y notificación de los incidentes y los errores causales que pongan en riesgo al paciente. La terminología se debe apegar al «Marco conceptual para la seguridad del paciente» de la Organización Mundial de la Salud' y al documento "Acciones esenciales para la seguridad del paciente». ${ }^{4}$ Además, tener una terminología armonizada facilitará la investigación y aprendizaje de los errores en favor de la seguridad del paciente. Durante el proceso también es importante contemplar el impacto de cada incidente sobre la salud física y psicológica de los pacientes, a pesar de no tener una consecuencia grave derivada del mismo.

Finalmente, la educación en el uso de dispositivos médicos de venta libre es un reto importante de la industria de dispositivos médicos, ya que los usuarios menos capacitados en el uso de los mismos podrían no percatarse del fallo del dispositivo o realizar un uso inapropiado del mismo poniéndose en ambos casos en riesgo su salud y que podría no 
notificarse a las unidades de tecnovigilancia, bloqueando así la mejora de la seguridad del paciente.

\section{AGRADECIMIENTOS}

Investigación financiada por el Prof. Eduardo Mucito-Varela.

\section{BibliografíA}

1. Organización Mundial de la Salud. Más que palabras. Marco Conceptual de la Clasificación Internacional para la Seguridad del Paciente Informe Técnico Definitivo Enero de 2009. OMS. 2009, pp. 1-160. Disponible en: http://www.who.int/patientsafety/implementation/icps/ icps_full_report_es.pdf.

2. Yu A, Flott K, Chainani N, Fontana G, Darzi A. Patient Safety 2030. London, UK: NIHR Imperial Patient Safety Translational Research Centre, 2016. Available in: http:// www.imperial.ac.uk/media/imperial-college/institute-ofglobal-health-innovation/centre-for-health-policy/PatientSafety-2030-Report-VFinal.pdf.

3. Secretaría de Salud. Norma Oficial Mexicana NOM241-SSA7-2012, Buenas Prácticas de Fabricación Para Establecimientos Dedicados a La Fabricación de Dispositivos Médicos. México: Diario Oficial de la Federación; 2012.

4. Consejo de Salubridad General. ACUERDO Por el que se declara la obligatoriedad de la implementación, para todos los integrantes del Sistema Nacional de Salud, del Documento Denominado Acciones Esenciales para la Seguridad del Paciente. México: Diario Oficial de la Federación; 2017.

5. Secretaría de Salud. Norma Oficial Mexicana NOM-240SSAT-2012, Instalación y Operación de La Tecnovigilancia. México: Diario Oficial de la Federación; 2012.

6. Comisión Federal para la Protección contra Riesgos Sanitarios. Farmacovigilancia en México. Gobierno de México; 2017. Disponible en: https://www.gob.mx/cofepris/ acciones-y-programas/farmacovigilancia-73541.

7. Comisión Federal para la Protección contra Riesgos Sanitarios. Información Referente a Tecnovigilancia 20142018. Gobierno de México; 2018. Disponible en: https:// www.gob.mx/cms/uploads/attachment/file/432576/ INCIDENTES_RELATIVOS_A_DISPOSITIVOS_M_DICOS_ CLASE_II_Y_III_RECIBIDOS_EN_EL_CNFV_DE_2014_A_ LA_FECHA_2.1.pdf.
8. Kumar RP. Latex allergy in clinical practice. Indian J Dermatol. 2012; 57 (1): 66-70. doi: 10.4103/0019-5154.92686.

9. Acham S, Truschnegg A, Rugani P, et al. Needle fracture as a complication of dental local anesthesia: recommendations for prevention and a comprehensive treatment algorithm based on literature from the past four decades. Clin Oral Investig. 2019; 23 (3): 1109-1119. doi: 10.1007/s00784-018-2525-8.

10. Vilos GA, Vilos AG. Weighted speculum buttock burn during gynecologic surgery. Obstet Gynecol. 2003; 101 (5, Part 2): 1064-1066. doi: https://doi.org/10.1016/S00297844(02)02331-7.

11. Angüiano-Sánchez NV, Perales-Quintana MM, Gabriel C, Cázares-Tamez R, Pérez-Chávez F, Llaca-Díaz JM. Errores en el laboratorio clínico; evaluación de tipos y frecuencias. Med Univ. 2011; 13 (52): 133-138.

12. Uppal V. An unrecorded pre-pre-analytical error in serum iron analysis. J Clin Diagn Res. 2015; 9 (17): 15376. doi: 10.7860/ JCDR/2015/15376.6749.

13. Park M, Won J, Choi BY, Lee CJ. Optimization of primer sets and detection protocols for SARS-CoV-2 of coronavirus disease 2019 (COVID-19) using PCR and real-time PCR. Exp Mol Med. 2020; 52 (6): 963-977. doi: 10.1038/s12276-0200452-7.

14. Njoroge SW, Nichols JH. Risk management in the clinical laboratory. Ann Lab Med. 2014; 34 (4): 274-278. doi: 10.3343/ alm.2014.34.4.274.

15. Mérida de la Torre FJ, Moreno-Campoy EE. Cestión de riesgos y mejora de la seguridad del paciente. In: Mérida de la Torre FJ, Moreno-Campoy EE, eds. Fundamentos de seguridad del paciente. Análisis y Estrategias en el Laboratorio Clínico. Madrid, España: Editorial Médica Panamericana; 2011, pp. 55-68.

16. Figueroa-Montes LE. Cestión de riesgos en los laboratorios clínicos. Acta Med Per. 2015; 32 (4): 247-250.

17. Secretaría de Salud. Norma Oficial Mexicana NOM007-SSA3-2011, Para la Organización y Funcionamiento de los Laboratorios Clínicos. México: Diario Oficial de la Federación; 2012.

18. NMX-EC-15189-IMNC-2015 (ISO 15189:2012). Laboratorios Clínicos-Requisitos particulares de la calidad y competencia. Instituto Mexicano de Normalización y Certificación, A.C., 2015.

19. Castellanos OF, Torres LM. Technology intelligence: methods and capabilities for generation of knowledge and decision making. PICMET '10 - Portl Int Cent Manag Eng Technol Proc - Technol Manag Glob Econ Growth. 2010; 1176-1184. 